\title{
Research and Design of Aging Suitability of Smart Bracelet Based on Implicit Human Computer Interaction
}

\author{
Jinmei OU, Gang LI ${ }^{\mathrm{a}, \mathrm{b}}$, and Qiuyu $\mathrm{LIU}^{\mathrm{c}}$ \\ ${ }^{a}$ College of Art, Chongqing University, Chongqing, China \\ ${ }^{\mathrm{b}}$ College of Humanities and Social Sciences, City University of Macau, Macau \\ ${ }^{ }$Department of Art and Product Design, Yibin University
}

\begin{abstract}
In order to explore a smart bracelet design strategy that is more suitable for the elderly, this paper studies relevant literatures and related products of smart bracelet at home and abroad, and sorts out basic concepts and related technologies of implicit interaction theory. Through questionnaire survey and semi-structured interview, we summarized. the basic characteristics of the elderly and the corresponding pain points of using smart wristband. We built a smart bracelet design scheme for the elderly based on implicit interaction theory, which provides certain theoretical support and practical reference value for the follow-up study on the agesuitability design of smart bracelet.
\end{abstract}

Keywords. Smart bracelet, implicit human computer Interaction, aging suitability, elderly

\section{Introduction}

Due to the vigorous development of science and technology at home and abroad, various chips with high performance and low power consumption have been launched one after another. More and more smart bracelet has gradually appeared in the eyes of the public. This type of device generally has the function of monitoring physiological parameters such as eye movement, heart rate, blood oxygen saturation, blood pressure, temperature, ecg and other parameters, and transmits the monitoring information to the corresponding APP in real time, thus providing reliable reference for human health management. However, the existing smart bracelet design on the market hardly takes into account the special needs of the elderly people, but mostly targets the young group. The aging problem is becoming increasing serious in most developing and developed countries. In the middle of this century, studies show that the proportion of elderly population over 60 years old in China will be as high as $37.8 \%$ [1]. Therefore, it is imperative to study the aging suitability design of smart bracelets. This paper attempts to discuss the design of smart bracelet for elderly people based on the basic characteristics of the elderly in physiology, psychology and cognition, combined with implicit interaction theory and corresponding key points. 


\section{Smart bracelet}

\subsection{Domestic and foreign literature research}

Smart bracelet is a digital monitoring device worn on the wrist with a corresponding mobile APP. It can objectively and continuously monitor physiological parameters and give intuitive and real-time feedback on activity [2]. With the growing maturity of mobile Internet technology, smart bracelet has gradually set off a wave of related research in academia in recent years. Searching in the Web of Science with "Smart Bracelet" as the subject heading, more than 3,000 related articles were found, mainly in the fields of engineering, computer science, instrumentation, and telecommunications. Among them, the most cited articles mainly focus on smart bracelets. The reliability and validity of monitoring [3], the related technologies of smart bracelets [4], the application of smart bracelets in medical treatment [5], etc. Searching on How Net with "smart bracelet" as the subject term, it can also be found that related articles are basically concentrated in the fields of computer science, industrial economy, automation technology, telecommunications technology, etc., the most influential articles in these papers are the same as those mentioned above, basically focusing on the monitoring reliability and validity of smart bracelets [6], related technologies [7], medical applications [8] and other aspects. It is not hard to see that no matter at domestic or foreign, the researches on smart bracelets focus more on the engineering level, and only a few articles study smart bracelets from the perspective of humanistic needs.

\subsection{Research on related products at domestic and foreign}

With the continuous improvement of people's health awareness, smart bracelet has already received higher and higher market response. Merchants of different brands have squeezed into the smart bracelet market. After 2014, the basic situation of smart bracelets just like a hundred flowers blossom, and a hundred schools of thought contend. Nevertheless, the functions of the smart bracelet are largely identical but with minor differences. As shown in Table 1, whether it is the hot-selling Xiaomi bracelet, Honor bracelet, OPPO bracelet in the domestic market, or the Jawbone UP bracelet and Fitbit Charge smart bracelet from abroad, they are limited to basic functions such as heart rate monitoring, sleep monitoring, exercise recording and alarm clock, and are mainly applied in the field of sports and fitness, with serious homogeneity for young groups.

Table 1. Functions of some hot-selling smart bracelets at domestic and foreign

\begin{tabular}{cr}
\hline Product name & Product features \\
\hline Mi Band 6 & $\begin{array}{r}\text { Heart rate monitoring, blood oxygen monitoring, sleep monitoring, } \\
\text { exercise record, message notification, payment function, weather } \\
\text { forecast, music playback control, calendar reminder, alarm clock }\end{array}$ \\
Honor Band 6 & $\begin{array}{r}\text { Heart rate monitoring, blood oxygen monitoring, sleep monitoring, } \\
\text { exercise record, message notification, payment function, weather } \\
\text { forecast, music playback control, calendar reminder, alarm clock, } \\
\text { female menstrual cycle management }\end{array}$ \\
\end{tabular}


OPPO bracelet

Jawbone UP24 bracelet

Fitbit Charge HR bracelet
Heart rate monitoring, blood oxygen monitoring, sleep monitoring, exercise record, message notification, payment function, weather forecast, music playback control, calendar reminder, alarm clock

\section{Current Status of Research on implicit human computer Interaction}

\subsection{Theoretical basis of implicit human computer Interaction}

Implicit Human Computer Interaction (IHCI) was first proposed by Kaiyan in $1996{ }^{\text {[9], }}$ and it is an important research frontier in the field of interaction. The real definition of the term implicit human computer Interaction is Albrecht Schmidt of the University of Karlsruhe, Germany. He defines implicit human computer Interaction as: users do not need to pay too much attention to the interaction process itself in the process of using the device or system, because the device or system can actively identify and understand user behavior, and there is no need to spend time studying how the device or system is used. The device or system can automatically and implicitly execute the conclusion instructions after analysis [10]. Ju Wendy and others believe that: implicit human computer Interaction is to actively perceive the user's target intention as the information input of its implicit human computer Interaction without affecting the user's attention, on the contrary, the device has high initiative [11].

The domestic research on implicit human-computer interaction can be roughly divided into three categories, first, based on the analysis of implicit human computer Interaction characteristics, relevant models and frameworks are established to guide the design. For example, Ye Xiyong et al. established dynamic context model and ADL-DBN reasoning model through the analysis of implicit human computer Interaction system [12]. Second, the application of implicit human computer Interaction in a specific field was studied. For example, Wang Wei and Yao Yushuang summarized the design principles of wearable implicit human computer Interaction by analyzing typical application cases [13]. Thirdly, the algorithm and model of implicit human computer Interaction are proposed from the technical point of view. For example, Wang Guojian and Tao Linmi analyzed the requirements of implicit human computer Interaction for computing system and constructed distributed vision system [14].

\subsection{Related technologies of implicit human computer Interaction theory}

The key to implicit human computer Interaction is perception and reasoning, which inevitably needs to talk about technical support. In this interactive mode, the system collects basic data through wearable devices, smart devices, smart phones, medical equipment, and other Internet of Thing' s devices, and then performs data annotation, data cleaning, data classification, data fusion, etc. Preprocessing, forming dynamic context information as the input of implicit human computer Interaction, and finally doing data analysis through deep learning, machine learning, data mining, regression analysis, small sample learning, etc., and to make reasonable inference to the user's 
intention, form the output of implicit human computer Interaction. The main technical support involved is shown in Table 2.

Table 2. The main technical support involved in implicit human computer Interaction

\begin{tabular}{ccc}
\hline Basic data collection & Data preprocessing & Data analysis \\
\hline Wearable device & Data annotation & Deep learning \\
Smart equipment & Data cleaning & Machine learning \\
Smart phone & Data classification & Data mining \\
Medical equipment & Data mix & Regression analysis \\
Other IOT devices & $\ldots \ldots .$. & Small sample learning \\
$\ldots . .$. & & $\ldots \ldots$
\end{tabular}

\section{Investigation and analysis of the basic characteristics of the elderly people and the key points of using smart bracelets}

Based on the convenience sampling method, this study between July and September 2021 in Chongqing city community, parks, supermarkets, markets, nursing homes and other places to choose aged more than 60 years of age, mental state, good communication skills and good life take care of the elderly people as the research object, 100 questionnaires, eventually recycling effective questionnaire 92 , effective rate was $93.3 \%$. And five elderly people were selected to experience the smart bracelet free of charge for 7 days through the purpose sampling method, and then conduct a semi-structured interview according to the usage situation. The basic information of the questionnaire subjects is shown in Table 3, and the basic information of the interview subjects is shown in Table 4.

Table 3. Basic information of questionnaire subjects

\begin{tabular}{cccc}
\hline Project & Group & Frequency & Composition ratio \\
\hline Gender & Male & 50 & $55.4 \%$ \\
& Female & 42 & $45.6 \%$ \\
Age & $60-70$ & 62 & $67.4 \%$ \\
& $71-80$ & 30 & $33.6 \%$ \\
\hline
\end{tabular}

Table 4. Basic information of interviewees

\begin{tabular}{|c|c|c|c|c|}
\hline Name & Gender & Native place & Age & Profession \\
\hline Mr. Wang & Male & Chongqing & 62 & $\begin{array}{c}\text { College and } \\
\text { university teacher }\end{array}$ \\
\hline Ms. Jiang & Female & Guangdong & 66 & Company employee \\
\hline Mr. Lee & Male & Liaoning & 61 & $\begin{array}{l}\text { College and } \\
\text { university } \\
\text { administrators }\end{array}$ \\
\hline Ms. Huang & Female & Hunan & 65 & $\begin{array}{l}\text { Middle school } \\
\text { teacher }\end{array}$ \\
\hline Mr Liang & Male & Sichuan & 69 & Company employee \\
\hline
\end{tabular}

\subsection{Survey results of the basic characteristics of the elderly people}

The results of the survey show that the basic characteristics of the elderly people will change according to their age, which can be roughly divided into three aspects :(1) in the physiological aspect, the elderly's vision, hearing and feeling will deteriorate with age, and their perceptual ability will decline; at the same time, relative to the young, the elderly people tendons, ligaments and other parts will atrophy, muscle volume, bone content reduction, flexibility, balance ability, muscle strength level are degraded to a 
certain extent, and then lead to the elderly people decreased mobility.(2) In terms of psychology, due to the change of roles and the decline of physiological functions, the elderly are prone to anxiety; and with the decline of physiological and cognitive functions, the elderly have many inconveniences in their lives. For example, the ability to accept new things is low, and the ability to deal with things is also low, which is very easy for the elderly to fall into negative feelings of self-criticism.(3) In terms of cognition, as older people age, their brains shrink to varying degrees, reducing the flexibility of cortical nerves, memory and thinking skills, and thus learning and hands-on skills.

\subsection{Survey results of key points in the use of smart bracelets for the elderly people}

Among the 92 valid questionnaires collected, only 8 elderly people have used smart bracelets, accounting for less than $10 \%$, but $54.3 \%$ of the elderly people expressed their willingness to try smart bracelets, therefore, the study on the aging design of smart bracelet not only has certain academic significance but has certain commercial value. In the subsequent semi-structured interviews, the focus is on the key points of use. According to the description of the interviewees, the key points of the elderly people in using the smart bracelet are as follows:

Table 5. The basic characteristics of the elderly people and the corresponding key points of using smart bracelets

\begin{tabular}{|c|c|c|}
\hline Basic features & & Corresponding key points \\
\hline \multirow[t]{2}{*}{$\begin{array}{l}\text { Physiological } \\
\text { characteristics }\end{array}$} & Decreased perception & $\begin{array}{l}\text { Difficult to perceive feedback information } \\
\text { accurately }\end{array}$ \\
\hline & Reduced mobility & $\begin{array}{l}\text { It is difficult to accurately implement specific } \\
\text { gesture interactions }\end{array}$ \\
\hline \multirow[t]{2}{*}{$\begin{array}{l}\text { Psychological } \\
\text { characteristics }\end{array}$} & Easy to feel anxious & $\begin{array}{l}\text { It is difficult to deal with complex interaction } \\
\text { processes }\end{array}$ \\
\hline & Nostalgia & It's hard to accept new versions, new styles \\
\hline \multirow[t]{3}{*}{$\begin{array}{l}\text { Cognitive } \\
\text { characteristics }\end{array}$} & Decreased thinking ability & $\begin{array}{l}\text { It's difficult to receive professional content about } \\
\text { parameters such as heart rate and blood pressure }\end{array}$ \\
\hline & Memory decline & $\begin{array}{l}\text { It's difficult to deal with complex interaction } \\
\text { processes }\end{array}$ \\
\hline & $\begin{array}{l}\text { Decline in learning and } \\
\text { operating ability }\end{array}$ & It's difficult to use complex APP functions \\
\hline
\end{tabular}

\section{Design strategies based on the theory of implicit human computer Interaction}

According to the results of the user survey above, a new design strategy can be obtained by combining the implicit interaction theory with the basic characteristics and corresponding pain points of the elderly group, which is specifically explained as follows.

\subsection{Perceived contextual information}

Interaction can be divided into two aspects: input and output. In Table 5, it is difficult to accurately implement specific gesture interactions due to reduced mobility, difficult to cope with complex interaction procedures due to anxiety and memory decline, and difficult to use complex APP functions due to decreased learning and operating capabilities, both focus on the information input during the interactions with bracelet. Implicit human computer Interaction is a new type of human-computer interaction under 
a human-centered computing model, one of its key elements is contextual information, which emphasizes the use of contextual information as interactive input to greatly improve the communication efficiency between human and system. Physiological parameter monitoring apps can be equipped with the perception of contextual information, and data processing can be guided by the use of contextual information, highlighting the focus of processing, and taking the initiative to provide corresponding services, on the one hand, processing speed can be accelerated, and on the other hand, the burden of the elderly can be greatly reduced. Specifically, the context information mainly includes the following two categories. 1. User context information, namely user behavior habits, historical experience, and physiological state, etc.2. Environmental contextual information, namely geographic location, physical environment, etc. There is no doubt that contextual information is key for systems to overcome the word-meaning gap between low-level sensor data and a high-level understanding of the user's intentions ${ }^{[23]}$.Accurately perceive contextual information, so that the information "input" of the smart bracelet no longer depends on the precise instructions of the elderly people, but the system actively collects user information and environmental information, and integrates user behavior, expression changes, voice tone, historical operations, Geographic location, temperature, humidity, etc. are used as the "inputs" of its implicit human computer Interaction. In studies by Xiyong Ye et al., for example, based on the implicit interaction theory and the system designed in combination with the elderly care examples, the system can recognize the actions of the elderly and use the detected data as input, when the system determines that the elderly fall abnormally, the system can directly and automatically send alarm information to the elderly service center through the network, without requiring the elderly to provide additional input to the system ${ }^{[12]}$.

\subsection{Reasonably infer user intentions}

Another key element of implicit human computer Interaction is to reasonably speculate on user intentions. The user is the main body of the interaction, only when the user's purpose and intention are realized, can the interaction process have value and significance. According to the user's contextual information and environmental context information perceived by the system, the senile's intention can be reasonably predicted, which is the basis for implicit interaction design to be integrated into aging suitability design. Difficulties in accurately perceiving feedback information caused by the decline in perception ability of middle-aged and elderly people mentioned by table 5 are all related to the aspect of the information input of the interaction. Most studies focus on how to make the elderly better "understand" the APP when dealing with the interactive output of the elderly. For example, in terms of the weak perception of the elderly, the strategy is to increase the font size and improve the color contrast. The implicit human computer Interaction emphasizes the initiative of the device, and the implicit human computer Interaction into the aging suitability design of the APP can make the APP more "understand" the elderly people. From the perspective of implicit human computer Interaction, the system reasonably infers the user's potential purpose based on the context information perceived by intelligent bracelet. It is of great significance for the elderly group to hide inefficient or repetitive tasks in the process of realizing their goals and reduce meaningless explicit interactions. In apps such as Alipay and WeChat, for example, when a user clicks the page of recharge, the system will reasonably predict the phone number that the user wants to recharge, and then automatically fill in the phone number for the user based on the user's historical recharge record so as to reduce the 
user's repeated input of the number. This can effectively reduce the user's operating burden and memory burden. Similarly, in the application of smart bracelets, the system can reasonably predict the intentions of the elderly by combining contextual information, and actively output the content and functions the elderly want to see.

\section{Design scheme of smart sleep aid bracelet for the elderly people based on implicit human computer Interaction}

\subsection{Overall system framework design}

The smart wristband for the elderly based on implicit interaction mainly consists of wristband hardware and APP software. A variety of sensors built into the bracelet can monitor the elderly's eye movement, heart rate, temperature, behavior, pulse, oxygen saturation and other data in real time and age, gender, medical history and other information of the elderly need to be input into the accompanying APP. Intelligent bracelet system based on implicit interaction requires semantic analysis of monitoring data and context information in order to obtain accurate situational judgment. The BERT deep learning model proposed by Google is composed of multi-layer bi-directional Transformer decoder, which can learn multi-level context information and better obtain context semantics. After optimization and modification, BERT model can be widely used in many fields, and can also be used as a basic model for context data analysis and intention inference in implicit interaction [15].

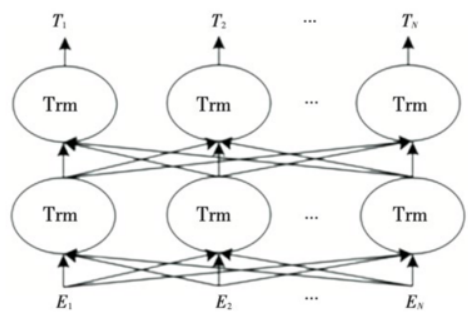

Figure 1. The basic model of BERT

The overall system framework design is shown in Figure 2. It consists of three parts : (1) Context information collection. A variety of sensors are used to collect human body data and environmental data as sources for constructing contextual data information. (2) Infer the user's purpose. Feature data such as human body and environment are extracted, and the intelligent system is trained by BERT model, so that the sensing data is connected with the user and the situation. Finally, the predicted user intention is output through deep learning model calculation. (3) Display and feedback. Display information in a way that is more consistent with the basic characteristics of older people based on reasonably inferred user intentions. 


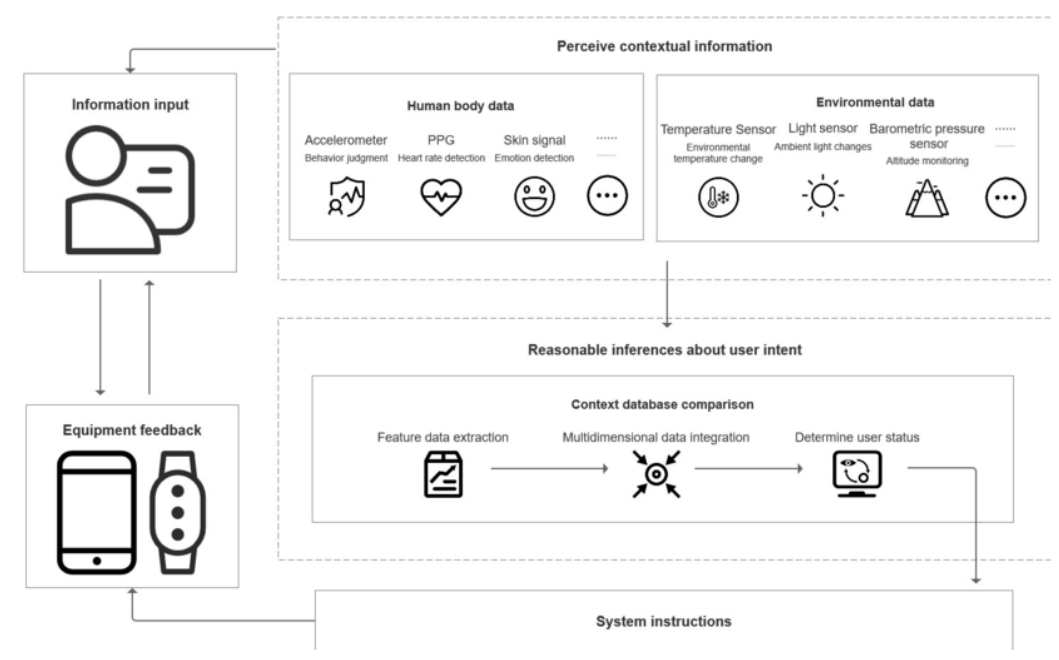

Figure 2. The Overall design framework of the intelligent bracelet

\section{2 basic function design}

Combined with relevant products in the market and the demand characteristics of the elderly, six basic functions of smart bracelet are proposed: (1) Emergency rescue. When the smart bracelet detects abnormal behavior of the elderly and determines that the elderly have fallen, it will timely alarm and send the specific location of the elderly to the emergency contact person. (2) Physiological status monitoring and reminder. Physiological parameters such as heart rate, blood oxygen, blood pressure and body temperature of the elderly were monitored in real time, and relevant strategies were recommended to the elderly based on the monitoring data. (3) Exercise monitoring and reminder. Combined with the age of the elderly, medical history, physical condition, personal habits, etc., for the elderly to formulate a reasonable exercise plan, and remind the elderly to complete. (4) Remind the medicine. Users can customize the dosage, medication reminder time. (5) Sedentary remind. When the wristband senses that the elderly have not been up for a long time, it sends out a sedentary reminder. (6) Intelligent navigation. The elderly can set the itinerary information before going out, and the APP will provide intelligent navigation to avoid forgetting the destination and the journey home.

\subsection{Intelligent sleep aid interactive process design}

$28 \%$ of the elderly over 60 years old in foreign countries suffer from sleep disorders ${ }^{[16]}$, and the figure in China is as high as $41.2 \%$ [17]. At present, most smart wristbands on the market have the function of sleep monitoring, but do not have the corresponding function of helping sleep. Studies have shown that listening to the right kind of music can help older people shorten sleep latency and sleep longer [18]. Therefore, our smart bracelet design uses music therapy to help the elderly to reduce sleep disorders. The specific interaction process of sleep AIDS is shown in Figure 3. Usually, the sleep state is divided into wakefulness, light sleep and deep sleep. Taking the motion recorder as an example, the three-axis acceleration sensor can capture the motion change data of human 
body in $\mathrm{X}, \mathrm{Y}$ and $\mathrm{Z}$ directions. The user's sleep motion rhythm (BM) can be obtained by formula (1), and the user's sleep state can be determined according to BM value [19]:

$$
B M(t)=\sqrt{x(t)^{2}+y(t)^{2}+z(t)^{2}}
$$

The mobile APP will play lullabies for the elderly based on contextual information such as their age and preferences. At the same time, the bracelet continuously monitors the physiological parameters of the elderly, and automatically stops the music playing when the elderly is judged to have entered the sleep state. In addition, if older person waves hand actively during hypnosis, the system will consider this as an input information of implicit interaction and stop playing the music, because in daily life, waving hand is a sign of denial.

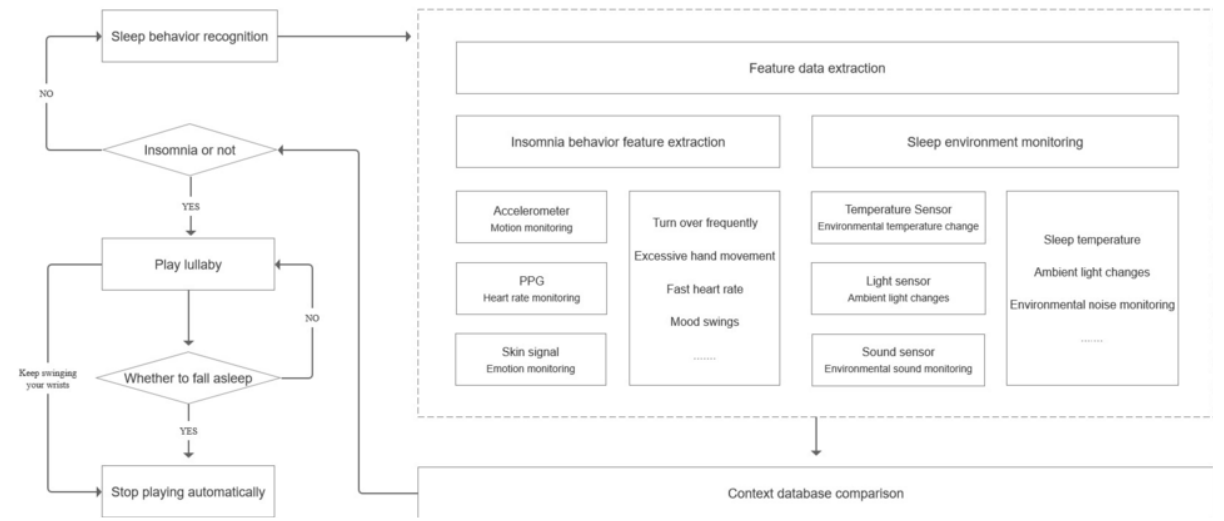

Figure3. Intelligent sleep aid interactive process design

\section{Conclusion and outlook}

Based on the status quo of smart bracelet and implicit interaction research, this paper sorted out the basic characteristics of the elderly people and the corresponding key points of using smart bracelet. At the same time, combined with the theory of implicit interaction, a new entry point for design was proposed. In the end, in order to improve the experience of using smart bracelet for the elderly, to reduce the operating burden, cognitive burden and memory burden in the process of using the elderly people, the design scheme of intelligent sleep aid bracelet for the elderly people based on implicit human computer Interaction is proposed, which also provides a new solution. In the AGE of 5G, science and technology will surely see further exponential development, how to better combine design and technology to enable the elderly to enjoy the modern life no different from the young, implicit human computer Interaction provides a good paradigm, will be the focus of future research on aging suitability. At the same time, since the core of implicit interaction lies in the perception and reasoning of contextual information, this inevitably involves certain privacy issues. In conclusion, the establishment of a reasonable user data privacy protection mechanism and the formulation of contextual information boundaries will be the focus of future research. 


\section{References}

[1] Du Peng, Li Long. Long-term Trends Projection of China's Population Aging in the New Era, Journal of Renmin University of China, 35 (2021), 1:96-109.

[2] Wen Xu, YUAN Bing, Li Hua, et al. The application of smart wearable devices in big data analysis of physical activity in China. China sports science and technology, 53 (2017), 2:80-87.

[3] Dong W, Zhang X, Liu X, et al. Evaluating the Consistency of Current Mainstream Wearable Devices in Health Monitoring: A Comparison Under Free-Living Conditions. Journal of Medical Internet Research, 19 (2017),3: e68.

[4] Yu S A, Hw A, Xc A, et al. High-efficiency self-charging smart bracelet for portable electronics ScienceDirect. Nano Energy, 55 (2019), 29-36.

[5] Marin I, Goga N. Model Checking the Properties of an Electronic Healthcare System to Facilitate the Detection of Preeclampsia through a Smart Bracelet // Model Checking the Properties of an Electronic Healthcare System to Facilitate the Detection of Preeclampsia through a Smart Bracelet. 2018.

[6] Hao Yingying, Zhang Lin, Ma Xiaokai, Cao Zhenbo. Journal of Shanghai University of Sport, 43 (2019), 4:73-83.

[7] Wang S D. Gesture recognition system of smart wristband based on inertial sensor. Jilin University,2016.

[8] Dong Zhuangzhuang, Zhang Yuting, Huang Yuwei, Zhang Yupeng, Long Sunhan, ZHANG Kaiyu, Tan Xiaodong. Application of smart bracelet in blood pressure monitoring of hypertension patients. China health industry, 16 (2019), 28,177-179.

[9] N. Kaiyan, Exploratory study of implicit theories in human computer interaction, Proceedings Sixth Australian Conference on Computer-Human Interaction, 1996, pp. 338-339.

[10] Schmidt A. Implicit Human Computer Interaction Through Context. Personal Technologies, 4 (2000) 2, 191-199.

[11] Leifer. The Design of Implicit Interactions: Making Interactive Systems Less Obnoxious. Design Issues, 2008.

[12] Ye Xiyong, Tao Linmi, Wang Guojian, Implicit interaction based on action understanding, in Proceedings of the 7th harmonious human-computer environment joint conference (HHME2011), Beijing, 2011, pp. 92-99.

[13] Wang Wei, YAO Yushuang. Analysis on Implicit Interaction Design of Wearable Products. Decoration, 06 (2016), 106-107.

[14] Wang Guojian, TAO Linmi. Distributed Vision System for Implicit Human Computer Interaction, Journal of image and graphics, 15 (2010),8,1133-1138.

[15] Devlin J, Chang M W, Lee K, et al. BERT: Pre-training of Deep Bidirectional Transformers for Language Understanding. Proceedings of NAACL-HLT 2019, pp. 4171-4186

[16] Endeshaw YW, Yoo W. Association between social and physical activities and insomnia symptoms among community-dwelling older adults. J Aging Health, 28 (2016), 1073-89.

[17] Xiong Feng, Lai Yuqing, Tu Jiaxin, et al. Epidemiological characteristics of sleep disorders in the Chinese elderly: a meta-analysis, Chinese Journal of Evidence-based Medicine, 19 (2019), 4: 398-403. (In Chinese)

[18] Lai H L, Good M. Music improves sleep quality in older adults. Journal of Advanced Nursing, 53 (2010), 3, 134-144.

[19] Huang Xiangyue, Hu Zhaoyan, Luo Yuzhou, Chen Zhenglong, Zhang Chunyuan, Liu Yuanyang. Method Research Analysis of Sleep Monitoring System and Its Classification Modes, Progress in biomedical engineering, 40 (2019), 1, 16-20+39. 